\title{
Pascale-Anne Brault
}

\author{
(Bard Collège, Annondale-on-Hudson, New York)
}

\section{(A) versions, inversions et subversions}

\section{Lecture du chapitre IV - Thomas l'Obscur, de Maurice Blanchot}

Parce que nous avons affaire à un texte qui n'est que dans la mesure où il n'est pas, un texte qui glisse, fuit et se disperse dès lors qu'on y appose son regard, toute tentative de lecture semble par avance vouée à l'échec. Et pourtant, nous ne pouvons nous empêcher, en tant que lecteurs, de nous trouver dans la même situation que Thomas lisant:

Il était, auprès de chaque signe, dans la situation où se trouve le mâle quand la mante religieuse va le dévorer. L'un et l'autre se regardaient (1950: 27).

C'est ce même regard qui, vibrant sur la marge tremblée entre vie et mort, nous attire dans le texte, nous séduit à notre insu et de façon irrémédiable. Ce regard cependant échappe à toute définition, car d'échange qu'il semble être au départ, 1l est modulé par un rapport de forces, le signe se dégageant de sa neutralité première et acquérant un pouvoir d'appropriation qui abolit l'échange: "il fut aperçu par l'intime du mot ... déjà les mots s'emparaient de lui et commençaient de le lire" (28). Ce rapport de forces mis en place, il ne sera plus fatt mention dans le texte de "regard" et seul le couple "oeil/yeux" va s'immiscer dans la trame du texte, le rhythmant de ses répétitions (dans ce seul chapitre, dix-neuf expressions se réfèrent au domaine de l'occulaire).

Ce passage du regard à l'oeil, du côté des choses au côté du corps (Préli: 1977. 83), de l'échange au pouvoir, s'effectue de façon progressive, quasi imperceptible, si bien que ce n'est pas tant une négation de l'un par l'autre qui nous est proposée mais bien plutôt, un passage obligé, une étape nécessaire car porteuse de mort et de vie, mais au demeurant non dépourvue de plaisir. Thomas "se voyait avec plaisir 
dans cet oeil qui le voyait" (28). Au delà de l'échange, c'est tout d'abord le glissement du texte en miroir qui s'opère. Miroir qui est déjà plus qu'un miroir, dans la mesure où Thomas ne peut se voir que parce qu'il est vu. Et pourtant, cette dialectique de "l'être vu" dont Sartre nous offre une version fort convaincante ( $L$ 'Etre et le néant. 1943: 317 et sq.) est moins un mouvement d'appropriation pure qu'une expérience d'éblouissement (Collin: 1971, 108).

Bien que le regard se manifeste dans les regards, bien qu'il soit une expérience dont attestent les récits, il est révélation d'un espace sans points de vue plutôt que d'un espace décentré par la multiplicité des points de vue. Ainsı le regard n'est pas fondamentalement mouvement du voir et de l'être vu, fût-il réciproque, va-et-vient du sujet à l'objet et de l'objet au sujet qui finit par définir ce dernier comme un "sujet-objet". Voir c'est éprouver dans la vision, laveuglement (Collin: 1971, 108-9).

Cette vie de l'oell dont Thomas ignore encore la menace potentielle d'aveuglement qu'elle représente, vivre dans le texte telle l'expérience d'une limite et, par le biais de la narration, nous avertit du retournement à venir du texte qui d'appropriable qu'il aurait pût être, finit par s'approprier de l'appropriant en puissance qu'est Thomas. Ainsi, tandis que Thomas "croyant être un lecteur profond" (28), est absorbé dans une tentative d'interprétation ou d'information signifiante, entrant:

avec son corps vivant dans les formes anonymes des mots, leur donnant sa substance, formant leurs rapports, offrant au mot être son être (29).

le texte est déjà ailleurs, nullement "complice" (28), nullement victime. C'est bien Thomas qui est "dévoilé" (29) et non le texte, mais il est dévoilé en tant qu'il est lui-même le texte, "il se reconnut avec dégoût sous la forme du texte qu'il lisait" (29). Dès lors, la limite entre l'objet et le sujet, le texte et Thomas, "le mot $I$ et le mot Je", frémit pour bientôt se dissoudre. Françoise Collin analysant L'Arrêt de mort insiste sur cette tension entre proximité et êloignement: 
Il semble qu'en ces points du moins, le regard devienne dans l'oeuvre blanchotienne, plus originel que le dialogue, et qu'en lui, ultilement, le plus prochain soit en même temps le plus lointain. dans une familiarité qui n'estompe pas mais révèle à l'extrême, laaltérité, altérité (et familiarité) d'autrui, d'une part, altérité (et familiarité) de l'être même, d'autre part (Collin: 1971, 111).

Et puis. Au-delà ou peut-être au creux même de la familiarité et de l'altérité, une présence. "Cette présence" (29), nocturne, inconnue, indéterminable. Est-elle générée par le texte? Y est-elle apposée? S'y substitue-t-elle? Nous ne savons. Quelques indices nous sont cependant offerts quant à sa nature. C'est une présence qui est par contradiction ou par inversion:

autant il êtait sûr qu'il n'y avait personne dans la chambre et même dans le monde, autant ll était sûr que quelqu'un était là (29).

à la fois "autour de lui et en lui" (30). Présence qui est aussi par son absence, présence qui est simultanément extérieure et intérieure et qui aveugle Thomas au fur et à mesure qu'il en prend conscience. Cette absence que Starobinski assimile dans Thomas l'Obscur à la transcendance absolue (in Collin: 1971, 112), est nuancée par Collin de la façon suivante:

si le Regard peut donc être lié à l'idée de Transcendance, c'est qu'il est rappel de l'inorigine. Cette transcendance, qu'il serait plus prudent d'appeler altérité, n'est pas la position d'un transcendant mais le rappel du caractère de non-identité du sujet, de sa non-adéquation avec soi, et à la limite de son a-subjectivité. Comme tel, ce Regard n'est donc pas lié à des yeux qui regardent: il est un regard aveugle dyeux absents (1971, 112).

Pour Thomas au sein du texte, le "regard aveugle" est vécu comme: "une modulation dans ce qui n'existait pas, une manière différente d'être absent, un autre vide dans lequel il s'animait" (30). La tension produite par cet espace 
'oxymoronique' est précisément la condition nécessaire à la possibilité-impossibilité d'une présence qui s'abolit et se recrée dans un même mouvement, tout en se modifiant à la fois. Car, de même que le signe au dêbut du chapitre se voyait investi d'un certain pouvoir, cette présence, peut-être de par son inéluctabilité, devient une "puissance" que Thomas s'évertue en vain de repousser. Elle est le signe annonciateur de la scission qui s'annonce sans toutefois se produire, précisément parce que lui, Thomas,

se sentait toujours plus proche d'une absence toujours plus monstrueuse dont la rencontre demandait l'infini du temps (31).

Un ultime renversement va être actualisé par le biais du dédoublement de Thomas, par le biais de son "non-adéquation à sol".

Attente et angoisse si insupportables qu'elles le détachèrent de lui-même. Une sorte de Thomas sortit de son corps et alla au devant de la menace qui se dérobait (31).

C'est grâce à ce dédoublement que Thomas opère le passage de l'espace au temps, du physique au métaphysique, pour finalement succomber à la force d'attraction qu'exerce cette puissance extérieure et retomber, de façon brutale, dans le domaine du physique. Ce que le dédoublement permet peut-être, c'est l'ouverture d'un interstice annonciateur de la lutte réelle. De la peur, de l'horreur, Thomas se meut vers le dénouement physique de cette situation. Comme le note Lucette Finas, son "agonie" (32) est bien à comprendre comme lutte, au sens grec du mot. Une lutte totale, un viol de tous les sens qui n'est pas sans rappeler les modifications subies par Grégoire dans La Métamorphose de Kafka. Et puis, le "point enflammé" (Bataille) du texte, le seul point-acte dont on puisse être vraiment certain:

C'est dans cet état qu'il se sentit mordu ou frappé, il ne pouvait le savoir, par ce qui lui sembla être un mot, mais qui ressemblait plutôt à un rat gigantesque, aux yeux perçants, aux dents pures. et qui était une bête toute-puissante (32). 
L'attaque, à mi-chemin entre le coup et la morsure, entre le réel et le fantastique, est aussi contrecarrée par la réaction inattendue de Thomas qui y résiste par désir plutôt que par peur. "Il se jeta sur elle et, lư enfonçant les ongles dans les entrailles, chercha à la faire sienne" (32). Cela donc qui, dans sa matérialité, s'apprête à commettre l'acte déterminant d'appropriation (la bête), se voit soumis à un acte d'appropriation plus puissant, plus rapide, comme si Thomas, au fil du texte, avait fait siennes les caractéristiques de la présence extérieure. C'est ce renversement de l'appropriation ou son inversion en son contraire, qui confère à la lutte sa "dignité". Thomas, en cherchant à se faire un avec la bête, en acquiert le comportement et les attitudes et cela, ajouté au flou d'un texte qui toujours nous échappe, fait qu'on ne sait plus très bien si "l'être couché par terre qui grinçait des dents, se labourait le visage, s'arrachait les yeux" (33) est Thomas ou la bête elle-même. A ce passage fait écho Derrida qui, à propos de Blanchot et des effets en forme de "ravage absolu", de "rapt", de "rature instantanée" de son texte, dit:

Je n'entends plus que des rats, le mot ou la syllabe qui viennent racler au fond de la gorge, la mort ou la jouissance résistant à elles-mêmes dans le râle. La figure du rat dans son bestiaire, et du rat dans la bouche, tous ces mots en ra dans Thomas $L^{\prime} O b$ scur et ailleurs... (Derrida: 1986, 92).

Au delà du grincement et du râle, c'est l'oeil qui, une fols de plus, fait son apparition, au creux même de sa négation possible. Le voir, dans ce chapitre, semble être intimement lié au savoir-non-savoir. Ce rapport obscurci et ténu à l'entendement est un rapport essentiel comme le souligne Blanchot dans sa référence à Heidegger et à son livre, Le Principe de raison:

Il [Heidegger] dit qu'entendre, c'est saisir par la vue, entrer dans le voir; 'dire dans la pensée grecque signifie amener à paraitre, faire apparaitre une chose dans la figure qui lui est propre, la montrer dans la manière dont elle nous regarde et c'est pourquoi, la disant, nous voyons clair en elle.' ... Cela correspond à l'idée que 'l'être est luisance'. D'où le privilège démesuré à la vue: privilège qui est originairement et implicitement supposé non seulement par toute métaphysique, mais par toute ontologie (et inutile de l'ajouter, toute phénoménologie) et selon lequel tout ce 
qui se pense, tout se ce qui se dit a pour mesure la lumière ou labsence de lumière (Entretien infint, 33-34).

Le texte débute sur un regard pour se voir ensuite parsemé d'yeux que l'on cherche à arracher et qui finalement constituent "l'opacité" du corps de Thomas. Que le corps de Thomas acquière ainsi une propriété normalement liée à la vision, nous donne presque l'impression que le texte tout entier se fond dans cet oell. Mais la qualité relativement négative que l'opacité peut avoir, traduit aussi, à un autre niveau, l'impossibilité ultime qu'il nous est faite de nous approprier le texte, l'ambivalence fondamentale d'une relation à l'entendement qui passe par "le voir". Car nous laissons Thomas à mi-chemin entre le cauchemar et l'explication du cauchemar, entre "l'impression reposante du sommeil" (32) et l'éveil, entre lu1même et l'autre, dans cet espace du fragmentaire qui peut-être "suppose, sollicite l'évacuation pure et simple du sujet" (Coulange: 1976, 119). Thomas, dans la mesure où l'on peut supposer qu'il aurait tenté de se poser ou de se définir comme sujet, n'est pas centre. Tout comme le texte, il n'est que dans la distance à lui-même, dans cette zone d'espacement qui le dissipe incontrôlablement.

What has happened? The 'I' has achieved what Emmanuel Levinas calls a difficult liberty, a break in participation with the lifeworld as world. We are on the brink of infinitude, since in the negativity of non-participation the difference between selfhood and otherness is given up, and intelligibility too gives way to a certain madness or disequilibration. Whatever holds the lifeworld together is by means of a difficult freedom unhinged and the subject vertiginously swept into an abysm or catastrophe of relations where no words serve to reobjectify or systematize what has been unloosened (Rappaport: 1987, 132).

Face à cet espace catastrophique, il ne nous est donné que ce jeu interminable et indéchiffrable entre yeux et regards, possession et dépossession, jeu qui interroge les notions traditionnelles de la limite et du visible. Ainsi que P. Adams Sitney le suggère, la prose de Blanchot n'est peut-être qu'un langage 
founded upon the value and truth of visibility, which has to perform contortions to create a space in which visibility can be examined and in which its unnamed alternatives and opposites can be propounded (1981: 194).

Ces contorsions, nous les retrouvons au niveau de toute l'oeuvre de Blanchot car finalement, c'est là l'essence même du littéraire pour Blanchot. L'oeuvre littéraire s'essaie et se cherche dans son impossibilité d'exister: elle tend vers son origine qui n'est autre que sa propre disparition. Voué à un recommencement perpétuel, le texte semble pris dans un mouvement circulaire, "reassessment éternel" qui (reconduit toujours à la conscience aigüe que le savoir est non-savoir. que le savoir/voir est aussi nécessairement l'impossibilité du savoir/voir. La question que soulève ce mouvement répétitif est celle de l'apport de son historicité. Y-a-t-il en effet un devenir, une évolution possible, une issue définitive qui permette l'éclatement ou la dispersion de ce mouvement? En fait, il semblerait que pour Blanchot la temporalité n'ait pour effet que de réitérer et d'épaissir (en quelque sorte) le mystère que constitue le lieu et la nature du centre, (réaffirmant en d'autres termes la distance incommensurable par rapport à l'être du texte. Ce processus signifie dans un même élan le découragement de toute interprétation car la réflexivité du texte est aussi le signe du renvol constant de l'interprétation à elle-même, et non à une vérité externe. C'est dans sa propre absence donc que le texte évolue, dans l'infidélité à un signifiant de lois qu'il se crée et se dé-crée, se crie et se décri(e)(t). Inscription dans sa propre négation, il est aussi sa propre mystification, sans cesse au bord de son auto-(d)énonciation, sans cesse toujours déjà érodé, oublié, condamné à son propre commencement. A la fois en dehors d'elle-même et sa matière propre, la parole blanchotienne vacille sans répit, dans le champ incertain de l'oubli, de la distance et de l'attente. Parler de vérité ou de réponse semble bien dérisoire dans un tel contexte. Dans une étude sur L'Innomable de Beckett, Blanchot suggère que

nous trouvons (la vraie réponse) plutôt dans le mouvement qui, à mesure que l'oeuvre cherche à s'accomplir, la ramène vers ce point où elle est à l'épreuve de l'impossibilité. Là, la parole ne parle pas, elle est, en elle rien ne commence, rien ne se dit, mais elle est toujours à nouveau et toujours se recommence. C'est 
cette approche de l'origine qui rend toujours plus menaçante l'expérience de l'oeuvre, menaçante pour celui qui la porte, menaçante pour l'oeuvre (1959: 316-7).

A l'ombre de cette menace, l'auteur du texte et son lecteur, de même que le texte lui-même, s'exposent à une perdition-mort sans cesse renouvelée, à un retour inévitable à leur silence respectif car, comme le souligne justement Derrida, "la digression de l'éloignement déroute, dès Thomas l'Obscur, toute logique de la limite, de l'opposition, de l'identité, de la contradiction" (1986: 47).

Ce sont ces contradictions internes (inversions de sens) qui font qu'il n'est pas possible d'avoir une version, une ligne de lecture dans Thomas l'Obscur. C'est tout comme si le texte exprimait une a-version, à la fois non-existence d'un sens unique et rejet-haine du texte comme texte, du lecteur comme lecteur. Ces mots-maux que nous lisons se reversant perpétuellement, se retournant sur eux-mêmes, finissent par devenir subversifs et rendent impossible la localisation ou identification de Thomas, du texte, du lecteur et de tous les autres éléments qui pourraient entrer en ligne de compte. En fin de compte, comme P. Adams Sitney le note, Blanchot ne nous ligue aucune doctrine, "but by making 'literature' less visible, more deadly, he challenges and thrills us with a theory of reading and writing as a solitary defense against the delusions of prefabricated clarity" (1981: 197). Il nous faut donc peut-être nous taire, et, maintenant, encore et toujours, nous laisser aller à la puissance du texte, de loeil du texte, nous y soumettre pour nous y trouver, nous y perdre et nous y retrouver.

Mais ce n'est pas certain.

\section{Bibliographie}

Blanchot, Maurice. L'Entretien infini. Gallimard: Paris, 1969

_. L'Espace littéraire. Gallimard: Paris, 1955.

—. Le Livre à venir. Gallimard: Paris, 1959. 
_. Thomas l'obscur. Gallimard: Paris, 1950.

Collin, Françoise. Maurice Blanchot et la question de l'écriture. Paris: Gallimard, 1971.

Coulange. Alain. "La dénomciation parcours: d'un arrêt dans la voix". In Lire Blanchot II., Pont-sur-Yvonne, France: Gramma 5, 1976.

Derrida, Jacques. Parages. Paris: Galilée: 1986.

Préli, Georges. La force du dehors: extériorité, limite et non-pouvoir à partir de Maurice Blanchot. Clamecy, France: Encres, éditions Recherches, 1977.

Rapaport, Herman. "Forecastings of Apocalypse: Asberry, Derrida, Blanchot". In Literature as Philosophy, Philosophy as Literature. Ed. Donald G. Marshall. Iowa City: University of Iowa Press, 1987.

Sartre, Jean-Paul. L'Etre et le néant. Paris: Gallimard, 1943.

Sitney, P. Adams. The Gaze of Orpheus par Maurice Blanchot. Traduit par Lydia Davis. Barrytown, N.Y.: Station Hill Press, 1981. 